\title{
Hub Angle Control for A Single Link Flexible Manipulator Based on Cuckoo Search Algorithm
}

\author{
Aiman Azrael Shaiful Nahar Sukri \\ School of Mechanical Engineering \\ College of Engineering \\ Selangor, Malaysia \\ aimanazraelsns@gmail.com
}

\author{
Annisa Jamali \\ Faculty of Engineering \\ Universiti Malaysia Sarawak \\ Sarawak, Malaysia \\ jannisa@unimas.my
}

\author{
Siti Sarah Zahidah Nazri \\ School of Mechanical Engineering \\ College of Engineering \\ Selangor, Malaysia \\ sarahzahidahnazri@gmail.com \\ Hanim Mohd Yatim \\ School of Mechanical Engineering \\ Universiti Teknologi Malaysia \\ Johor, Malaysia \\ hanim.my@utm.my
}

\author{
*Muhamad Sukri Hadi \\ School of Mechanical Engineering \\ College of Engineering \\ Selangor, Malaysia \\ msukrihadi@uitm.edu.my \\ Intan Zaurah Mat Darus \\ School of Mechanical Engineering \\ Universiti Teknologi Malaysia \\ Johor, Malaysia \\ intan@utm.my
}

\begin{abstract}
Flexible manipulators are one of the promising devices that can be applied in many fields especially in automation and manufacturing fields as they are designed to reduce energy consumption and increase the speed of operation. However, agitation process experienced in the complex structure of the system which causes unwanted vibration will affect the precision of operation. Thus, an efficient control system is required to make them functional. Therefore, the development of an accurate model of flexible manipulator was presented prior to establishing active vibration control to suppress the vibration and increase efficiency of the system. This paper presents the development of a Proportional-IntegralDerivative controller based on cuckoo search algorithm for a single link flexible manipulator system. Initially, the system was modelled using input and output experimental data of the hub angle. System identification was implemented via swarm intelligence algorithm known as cuckoo search algorithms based on auto regressive with exogenous model structure. Then, the performance of proposed algorithms was validated based on three robustness methods known as mean squared error, pole zero diagram stability and correlation tests. The simulation results showed superior performance of cuckoo search algorithm by achieving lowest mean squared error, good correlation tests and high root locus stability. Then, the cuckoo search model was implemented in the proposed control scheme with the aim of accurate positioning at the end point of flexible manipulator.
\end{abstract}

Keywords-flexible manipulator, swarm intelligence algorithm, cuckoo search algorithm, hub angle, pid controller

\section{INTRODUCTION}

With advances in material technology, flexible manipulator is highly demanded, especially in the robot areas due to its faster system response, less overall cost, manoeuvrable and safer operation. Besides that, it reduces the energy consumption since it used less powerful actuators [1]. The applications of flexible manipulator are vast such as in NASA Remote Manipulator System and satellites [2], manufacturing operations including vehicle assembly components, construction automation such as robotic excavators, cranes and so forth [3]. However, Qiu et. al. (2018) stated that, the downside of this structure is the vibration problem due to coupling effects, tough dynamic, highly nonlinear and low stiffness [4]. At high speed of motion, the vibration will become much worse, thus, leads to discomfort, noise, and disturbance to the surrounding which are undesirable and would have a negative effect in the industry such as higher overall cost, machine performances affected and structural fatigue [5].
Chen and his team (2018) mentioned that, the unwanted vibration will reduce the positioning accuracy and the control performances of single-link manipulators [6]. Vibration controls are then introduced to eliminate the undesired vibration. Modelling of flexible manipulator is done prior to obtain the best control. Theoretical models are crucial to be identified as it reflect the real behaviour and structural properties which is convenient for statistical analysis [7]. There are numerous modelling methods to solve major engineering problems, for instance, partial differential equation (PDE), finite difference method (FDM) and finite element method (FEM). However, a recent alternative and more accurate method that has been identified by researchers is system identification (SI) approach via swarm intelligence algorithm (SIA). The main concept of SI is to evaluate the compliance of current systems by recording the input-output vibrations of the structure due to vibration source in discrete time signals [8].

Various SIA have been developed, one of them is Cuckoo Search (CS) algorithm. It is nature-inspired algorithm, which describes cuckoo's searching behaviour based on parasitized breeding mechanism of cuckoo's egg and Levy flight search principle [9]. CS algorithm is one of the algorithms used to model structure to achieve optimum reduction of unwanted vibration. Zhang et al. (2019) said that its advantages and worthiness in producing the correct model structure will have an effective influence on system vibration reduction [10]. The objective of this study is to model a single-link flexible manipulator system based on parametric system identification method via CS algorithm by utilizing input-output data from the experimental study. The performance and efficiency of the developed model are validated with three robustness approaches known as mean squared error, correlation tests and pole zero diagram stability. The developed model is then implemented in proportional-integral-derivative (PID) control scheme with the aim of accurate positioning at the end point of flexible manipulator.

\section{EXPERIMENTAL SETUP For DATA COLLECTION}

In this study, experimental setup of flexible manipulator was crucial as to acquire vibration input-output data of hub angle. The flexible manipulator structure was made of single link thin aluminium alloy with the dimension of $600 \mathrm{~mm}$ in length, $40 \mathrm{~mm}$ in width and $1.5 \mathrm{~mm}$ in thickness. The flexible manipulator was tethered to one end of the motor and free on the other [11]. The properties of the system are described in Table 1. Data collection of the structural behaviour was accomplished using data acquisition system (DAQ) that can 